\title{
DISTRIBUIÇÃO MENSAL DOS PEIXES MAIS REPRESENTATIVOS DA LAGOA RODRIGO DE FREITAS, RIO DE JANEIRO, BRASIL
}

\author{
José V. Andreata ${ }^{1}$ \\ Alex G. Marca ${ }^{1}$ \\ Claúdio L. Soares ${ }^{1}$ \\ Rubens da S. Santos ${ }^{2}$
}

\begin{abstract}
MONTHLY DISTRIBUTION OF MOST REPRESENTATIVE FISH OF RODRIGO DE FreITAS LAGOON, RIO DE JANEIRO, BRAZIL. From the results obtained in four years of monthly fish sampling and salinity and temperature verification of Lagoa Rodrigo de Freitas lagoon, an analysis of the monthly capture distribution of the nine most representative species, Brevoortia aurea (Spix, 1829), B. pectinata (Jenyns, 1842), Genidens genidens (Valenciennes, 1839), Geophagus brasiliensis (Quoy \& Gaimard, 1824), Gerres aprion Cuvier, 1829, Jenynsia lineata (Jenyns, 1842), Mugil liza (Valenciennes, 1836), Poecilia vivipara (Bloch \& Schneider, 1801) and Xenomelaniris brasiliensis (Quoy \& Gaimard, 1824) was performed. The results show that Lagoa waters have an average salinity of $11.06 \%$ during the sampling period. Temperature showed its highest values from November to February and lowest values from June to August. G. brasiliensis, G. aprion, J. lineata, M. liza, P. vivipara e X. brasiliensis were constant during the 4 years. $B$. aurea showed highest frequency in year IV. B. pectinata was constant in years I and II and G. genidens showed only sparse captures.

KEY WORDS. Fishes, ecology, Rodrigo de Freitas Lagoon, Brazil
\end{abstract}

Os ambientes costeiros apresentam elevada produtividade biológica, sendo importantes para o recrutamento de várias espécies de peixes que as utilizam em todo ou apenas parte de seu ciclo de vida. Evidentemente podem ocorrer oscilações quantitativas na composição das espécies durante o ano, decorrentes de vários fatores, dos quais destacam-se os relacionados as estratégias reprodutivas e alimentares.

De uma maneira geral, esses ambientes apresentam dificuldades na renovação das águas e de assimilação de substâncias alóctones (MIRANDA 1984), sendo que as lagunas localizadas nas proximidades de áreas urbanas são as que mais sofrem degradação, principalmente pela urbanização mal planejada. Esta ação antrópica geralmente ocasiona modificações na geomorfologia destes ecossistemas, que muitas das vezes altera a circulação das águas e as trocas com o mar adjacente, o que afetará a biota local.

1) Instituto de Ciências Biológicas e Ambientais, Universidade Santa Úrsula. Rua Fernando Ferrari 75, 22231-040 Rio de Janeiro, Rio de Janeiro, Brasil. Bolsista do CNPq.

2) Departamento de Biologia Animal e Vegetal, Universidade do Estado do Rio de Janeiro. Rua São Francisco Xavier 524, 20550-013 Rio de Janeiro, Rio de Janeiro, Brasil. Bolsista do CNPq. 
Considerando-se os objetivos do presente estudo, verificou-se que a localização da Lagoa Rodrigo de Freitas favoreceu a sua degradação, que se iniciou com sucessivos aterros marginais (OlIVEIRA 1955; SALLES-ANDRADE 1973; PIRES 1977), visando a exploração pelo mercado imobiliário, tendo como conseqüência a modificação de sua linha de contorno original (BRITO \& LEMOS 1982) e a criação das ilhas artificiais Piraquê e Caiçaras (OliveIRA 1955), ocasionando uma perda considerável de superfície do espelho d'água, diminuição da profundidade e, conseqüentemente, da coluna d'água, além da redução da circulação e renovação das águas.

Este trabalho objetiva analisar as oscilações quantitativas mensais de captura das espécies de peixes mais representativas da Lagoa Rodrigo de Freitas durante $o$ período de março de 1991 a fevereiro de 1995, relacionando estas oscilações com a salinidade, que é um dos fatores limitantes das espécies em uma laguna devido aos problemas osmóticos, e a temperatura que, provavelmente, caracteriza a sazonalidade local.

\section{MATERIAL E MÉTODOS}

A Lagoa Rodrigo de Freitas é uma laguna costeira urbana, de forma poligonal irregular, localizada na zona sul da cidade do Rio de Janeiro, nas latitudes $22^{\circ} 57^{\prime} 22^{\prime \prime}$ a $22^{\circ} 58^{\prime} 09^{\prime \prime S}$ e longitudes $43^{\circ} 11^{\prime} 09^{\prime \prime}$ a $43^{\circ} 13^{\prime} 03^{\prime \prime W}$. Possui três quilômetros de diâmetro em sua maior largura e 7,5Km de perímetro (OLIVEIRA 1976). A profundidade máxima de massa d'água é de cinco metros (SALLES-ANDRADE 1973). Verificou-se, porém, nas amostragens, profundidades de até 11 metros. Seu fundo é composto, em certas áreas, por vasa e, naqueles próximos às margens, especialmente nos pontos onde há descargas de águas pluviais e na embocadura do canal do Jardim de Alah, por areia e conchas de moluscos bivalves (ARAGÃo et al. 1939; FEEMA 1975).

A Lagoa é circundada pelos morros do Cantagalo, dos Cabritos e da Saudade, à leste; do Corcovado e Sumaré, ao norte, e pelos bairros de Ipanema e Leblon, ao sul. A ligação com o Oceano Atlântico ocorre através do canal do Jardim de Alah e o principal aporte de água doce ocorre pelo Rio dos Macacos (Fig. 1).

No período de março de 1991 a fevereiro de 1995, foram realizadas amostragens mensais dos peixes da Lagoa Rodrigo de Freitas, que foi dividida aleatoriamente em quatro áreas de coleta. A metodologia mensal utilizada para a captura dos espécimes foi a de ANDREATA et al. (1989, 1990a,b, 1992), que consiste na utilização de tarrafas, malhas de 15, 18 e $20 \mathrm{~mm}$ de entrenó e esforço de 30 tarrafadas por área; arrasto-de-praia, malha de $15 \mathrm{~mm}$ de entrenó com sub-saco de malha de $3 \mathrm{~mm}$ de entrenó e esforço de três arrastos marginais com 50m de extensão cada, por área; puçá, malha de $3 \mathrm{~mm}$ de entrenó e esforço de dois lances, de $50 \mathrm{~m}$ de extensão cada, por área, e rede-de-espera, malha de $35 \mathrm{~mm}$ de entrenó, deixada à deriva durante todo o período de coleta (aproximadamente quatro horas).

A identificação taxonômica foi baseada em FIGUEIREDo \& MENEZES (1978, 1980), FisCHER (1978), LEMA et al. (1979), MENEZES \& FigUEIREDO (1980, 1985), VOLCKER \& ANDREATA (1982), GODOY (1987) e WhiteHEAD et al. (1988). Na identificação dos Gerreidae utilizou-se os trabalhos de ANDREATA $(1988,1989)$. 
Os valores da salinidade, obtidos através refratômetro, e os da temperatura, por meio de termômetro de mercúrio comum, foram verificados nas quatro áreas de coleta, sendo usados, neste trabalho, os valores médios mensais.

Para efeito desta análise, o período amostrado foi dividido em Ano I, março de 1991 a fevereiro de 1992; Ano II, março de 1992 a fevereiro de 1993; Ano III, março de 1993 a fevereiro de 1994 e Ano IV, março de 1994 a fevereiro de 1995.

Na distribuição mensal de captura foram analisadas as espécies que obtiveram percentual de captura $\geq 1 \%$ em pelo menos dois anos de amostragem.

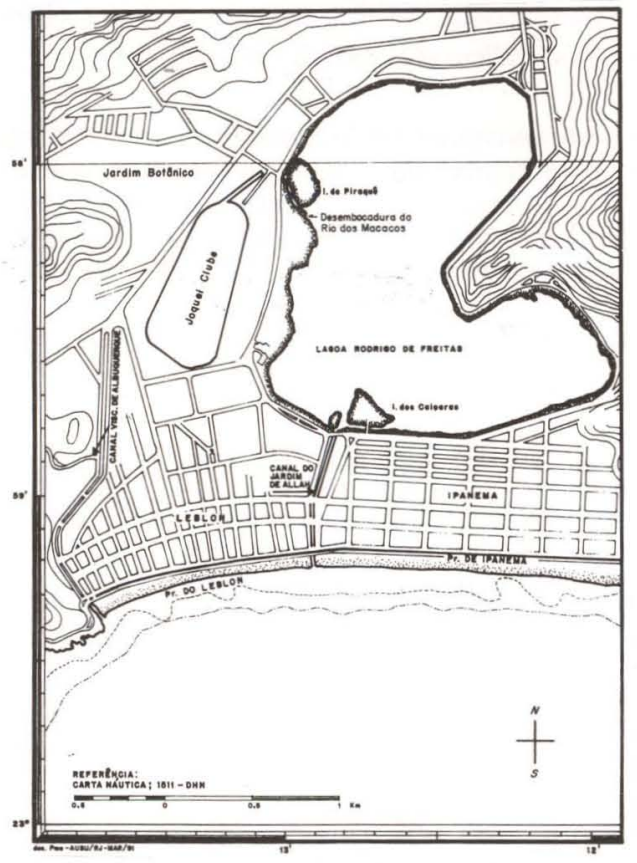

Fig. 1. Localização da área de estudo, Lagoa Rodrigo de Freitas, Rio de Janeiro.

\section{RESULTADOS}

A distribuição mensal dos valores médios da temperatura mostrou padrões esperados de oscilação, que refletiram a sazonalidade da região. Assim sendo, os meses do ano que correspondem o inverno no hemisfério sul, acrescidos dos meses adjacentes a esta estação do ano, apresentaram águas mais frias, em torno de 19 a $20^{\circ} \mathrm{C}$, enquanto que aqueles correspondentes ao verão e meses adjacentes, as águas apresentaram-se mais quentes, em torno de 25 e $30^{\circ} \mathrm{C}$ (Figs 2-10). Em relação à salinidade, verificou-se a tendência de padrões geralmente inversos aos da temperatura. Há notadamente dois períodos distintos em relação a este fator, sendo que o primeiro, entre junho de 1991 a agosto de 1992, apresentou médias variando da faixa de 13 a 23\%, enquanto que o segundo, entre setembro de 1992 a fevereiro de 
1995, a faixa de variação foi de 2 a $16 \%$. Em relação as três primeiras amostragens realizadas na região, entre março e maio de 1991, os valores médios de salinidade foram inferiores a $9 \%$, porém não há registros anteriores que possam ser acrescentados a estes resultados (Figs 2-10).

Durante os quatro anos amostrados, foram capturados 48.250 espécimes de peixes pertencentes a 55 espécies, listadas na tabela I. As espécies com maior percentual de captura foram Brevoortia aurea (Spix, 1829), Brevoortia pectinata (Jenyns, 1842), Genidens genidens (Valenciennes, 1839), Geophagus brasiliensis (Quoy \& Gaimard, 1824), Gerres aprion Cuvier, 1829, Jenynsia lineata (Jenyns, 1842), Mugil liza (Valenciennes, 1836), Poecilia vivipara (Bloch \& Schneider, 1801) e Xenomelaniris brasiliensis (Quoy \& Gaimard, 1824).

Tabela I. Listagem das espécies capturadas na Lagoa Rodrigo de Freitas no período de março de 1991 a fevereiro de 1995. Número de exemplares capturados e abundância relativa por ano de coleta e geral. (Ano I) março de 1991 a fevereiro de 1992, (Ano II) março de 1992 a fevereiro de 1993, (Ano III) março de 1993 a fevereiro de 1994, (Ano IV) março de 1994 a fevereiro de 1995, (AR) abundância relativa.

\begin{tabular}{|c|c|c|c|c|c|c|c|c|c|c|}
\hline \multirow{2}{*}{ Espécies } & \multicolumn{2}{|c|}{ Anol } & \multicolumn{2}{|c|}{ Ano II } & \multicolumn{2}{|c|}{ Ano III } & \multicolumn{2}{|c|}{ Ano IV } & \multicolumn{2}{|c|}{ AR } \\
\hline & $n^{\circ}$ & $\%$ & $n^{\circ}$ & $\%$ & $n$ & $\%$ & $n^{0}$ & $\%$ & $n^{0}$ & $\%$ \\
\hline Elops saurus & 14 & 0,13 & 31 & 0,27 & 19 & 0,23 & 29 & 0,16 & 93 & 0,19 \\
\hline Brevoortia aurea & 0 & 0,00 & 126 & 1,09 & 4 & 0,05 & 4838 & 27,42 & 4968 & 10,29 \\
\hline Brevoortia pectinata & 3393 & 31,71 & 1496 & 12,95 & 0 & 0,00 & 865 & 4,90 & 5754 & 11,92 \\
\hline Harengula clupeola & 0 & 0,00 & 0 & 0,00 & 2 & 0,02 & 3 & 0,02 & 5 & 0,01 \\
\hline Anchoa januaria & 0 & 0,00 & 38 & 0,33 & 0 & 0,00 & 0 & 0,00 & 38 & 0,07 \\
\hline Anchoa tricolor & 6 & 0,04 & 0 & 0,00 & 0 & 0,00 & 0 & 0,00 & 4 & * \\
\hline Anchoviella lepidentostole & 66 & 0,62 & 108 & 0,93 & 0 & 0,00 & 1 & 0,01 & 175 & 0,36 \\
\hline Genidens genidens & 524 & 4,90 & 131 & 1,13 & 1 & 0,02 & 0 & 0,00 & 656 & 1,33 \\
\hline Strongylura marina & 0 & 0,00 & 3 & 0,03 & 0 & 0,00 & 0 & 0,00 & 3 & $\star$ \\
\hline Phalloptychus januarius & 0 & 0,00 & 0 & 0,00 & 0 & 0,00 & 1 & 0,01 & 1 & * \\
\hline Poecilia reticulata & 14 & 0,13 & 42 & 0,36 & 16 & 0,19 & 0 & 0,00 & 72 & 0,15 \\
\hline Poecilia vivipara & 661 & 6,18 & 1107 & 9,58 & 2236 & 26,76 & 2743 & 15,55 & 6747 & 13,98 \\
\hline Xiphophurus helleri & 0 & 0,00 & 0 & 0,00 & 0 & 0,00 & 1 & 0,01 & 1 & * \\
\hline Jenynsia lineata & 831 & 7,77 & 979 & 8,47 & 1226 & 14,67 & 1599 & 9,06 & 4635 & 9,06 \\
\hline Xenomelaniris brasiliensis & 2947 & 27,54 & 4235 & 36,65 & 2383 & 28,52 & 5591 & 31,69 & 15156 & 31,41 \\
\hline Oostethus lineatus & 0 & 0,00 & 2 & 0,02 & 4 & 0,05 & 0 & 0,00 & 6 & 0,01 \\
\hline Syngnathus rousseau & 0 & 0,00 & 1 & 0,01 & 0 & 0,00 & 0 & 0,00 & 1 & $*$ \\
\hline Prionotus punctatus & 1 & 0,01 & 1 & 0,01 & 0 & 0,00 & 0 & 0,00 & 2 & $*$ \\
\hline Centropomus parallelus & 10 & 0,09 & 11 & 0,10 & 63 & 0,75 & 1 & 0,01 & 85 & 0,17 \\
\hline Centropomus undecimalis & 1 & 0,01 & 35 & 0,30 & 107 & 1,28 & 0 & 0,00 & 143 & 0,29 \\
\hline Pomatomus saltator & 1 & 0,01 & 0 & 0,00 & 1 & 0,01 & 3 & 0,02 & 5 & 0,01 \\
\hline Caranx latus & 22 & 0,21 & 36 & 0.31 & 0 & 0,00 & 5 & 0,03 & 63 & 0,13 \\
\hline Oligoplites saunus & 0 & 0,00 & 1 & 0,01 & 0 & 0,00 & 0 & 0,00 & 1 & $*$ \\
\hline Trachinotus carolinus & 3 & 0,03 & 3 & 0,03 & 2 & 0,02 & 1 & 0,01 & 9 & 0,02 \\
\hline Trachinotus falcatus & 6 & 0,06 & 1 & 0,01 & 2 & 0,02 & 0 & 0,00 & 9 & 0,02 \\
\hline Lutjanus analis & 0 & 0,00 & 1 & 0,01 & 0 & 0,00 & 0 & 0,00 & 1 & $*$ \\
\hline Diapterus lineatus & 0 & 0,00 & 0 & 0,00 & 103 & 1,23 & 1 & 0,01 & 104 & 0,21 \\
\hline Diapterus rhombeus & 0 & 0,00 & 14 & 0,12 & 27 & 0,32 & 0 & 0,00 & 41 & 0,08 \\
\hline Diapterus richii & 16 & 0,15 & 15 & 0,13 & 43 & 0,51 & 0 & 0,00 & 74 & 0,15 \\
\hline Gerres aprion & 696 & 6,51 & 393 & 3,40 & 105 & 1,26 & 47 & 0,27 & 1241 & 2,57 \\
\hline Gerres gula & 0 & 0,00 & 1 & 0,01 & 2 & 0,02 & 0 & 0,00 & 3 & 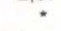 \\
\hline Gerres melanopterus & 4 & 0,04 & 0 & 0,00 & 9 & 0,11 & 7 & 0,04 & 20 & 0,04 \\
\hline Orthopristis ruber & 0 & 0,00 & 2 & 0,02 & 0 & 0,00 & 0 & 0,00 & 2 & $*$ \\
\hline Pomadasys croco & 0 & 0,00 & 1 & 0,01 & 0 & 0,00 & 0 & 0,00 & 1 & $*$ \\
\hline Micropogonias furnieri & 38 & 0,36 & 22 & 0,19 & 23 & 0,28 & 5 & 0,03 & 88 & 0,18 \\
\hline Archosargus rhomboidalis & 1 & 0,01 & 0 & 0,00 & 1 & 0,01 & 0 & 0,00 & 2 & * \\
\hline Diplodus argenteus & 0 & 0,00 & 0 & 0,00 & 0 & 0,00 & 1 & 0,01 & 1 & $*$ \\
\hline Chaetodiptenus faber & 0 & 0,00 & 3 & 0,03 & 1 & 0,01 & 0 & 0,00 & 4 & * \\
\hline
\end{tabular}


Tabela I. Continuação.

\begin{tabular}{|c|c|c|c|c|c|c|c|c|c|c|}
\hline \multirow{2}{*}{ Espécies } & \multicolumn{2}{|c|}{ Anol } & \multicolumn{2}{|c|}{ Ano II } & \multicolumn{2}{|c|}{ Ano III } & \multicolumn{2}{|c|}{ Ano IV } & \multicolumn{2}{|c|}{ AR } \\
\hline & $n^{\circ}$ & $\%$ & $n^{0}$ & $\%$ & $n$ & $\%$ & $n^{0}$ & $\%$ & $n^{\circ}$ & $\%$ \\
\hline Geophagus brasiliensis & 103 & 0,96 & 146 & 1,26 & 718 & 8,59 & 501 & 2,84 & 1468 & 3,04 \\
\hline Tilapia rendalli & 1 & 0,01 & 0 & 0,00 & 0 & 0,00 & 0 & 0,00 & 1 & * \\
\hline Mugil curema & 75 & 0,70 & 85 & 0,74 & 51 & 0,61 & 75 & 0,43 & 286 & 0,59 \\
\hline Mugil Igaimardianus & 11 & 0,10 & 19 & 0,16 & 0 & 0,00 & 0,00 & 0,00 & 30 & 0,06 \\
\hline Mugil liza & 1160 & 10,84 & 2407 & 20,83 & 1156 & 13,83 & 1245 & 7,06 & 5968 & 12,36 \\
\hline Mugil platanus & 17 & 0,16 & 2 & 0,02 & 1 & 0,01 & 1 & 0,01 & 21 & 0,04 \\
\hline Mugil trichodon & 0 & 0,00 & 1 & 0,01 & 0 & 0,00 & 0 & 0,00 & 1 & $*$ \\
\hline Dormitator maculatus & 1 & 0,01 & 5 & 0,04 & 14 & 0,17 & 0 & 0,00 & 20 & 0,04 \\
\hline Awaos tajasica & 4 & 0,04 & 5 & 0,04 & 2 & 0,02 & 0 & 0,00 & 11 & 0,02 \\
\hline Bathygobius soporator & 0 & 0,00 & 0 & 0,00 & 0 & 0,00 & 3 & 0,02 & 3 & $\star$ \\
\hline Gobionellus boleosoma & 0 & 0,00 & 0 & 0,00 & 0 & 0,00 & 1 & 0,01 & 1 & • \\
\hline Gobionellus oceanicus & 1 & 0,01 & 5 & 0,04 & 26 & 0,31 & 43 & 0,24 & 75 & 0,15 \\
\hline Microgobius meeki & 0 & 0,00 & 4 & 0,03 & 0 & 0,00 & 17 & 0,10 & 21 & 0,04 \\
\hline Paralichthys orbignyana & 2 & 0,02 & 1 & 0,01 & 1 & 0,01 & 0 & 0,00 & 4 & $\star$ \\
\hline Achirus lineatus & 69 & 0,64 & 34 & 0,29 & 5 & 0,06 & 15 & 0,09 & 123 & 0,25 \\
\hline Stephanolepis hispidus & 1 & 0,01 & 1 & 0,01 & 0 & 0,00 & 0 & 0,00 & 2 & $*$ \\
\hline Sphoeroides spengleri & 1 & 0,01 & 0 & 0,00 & 0 & 0,00 & 0 & 0,00 & 1 & * \\
\hline
\end{tabular}

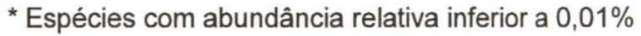

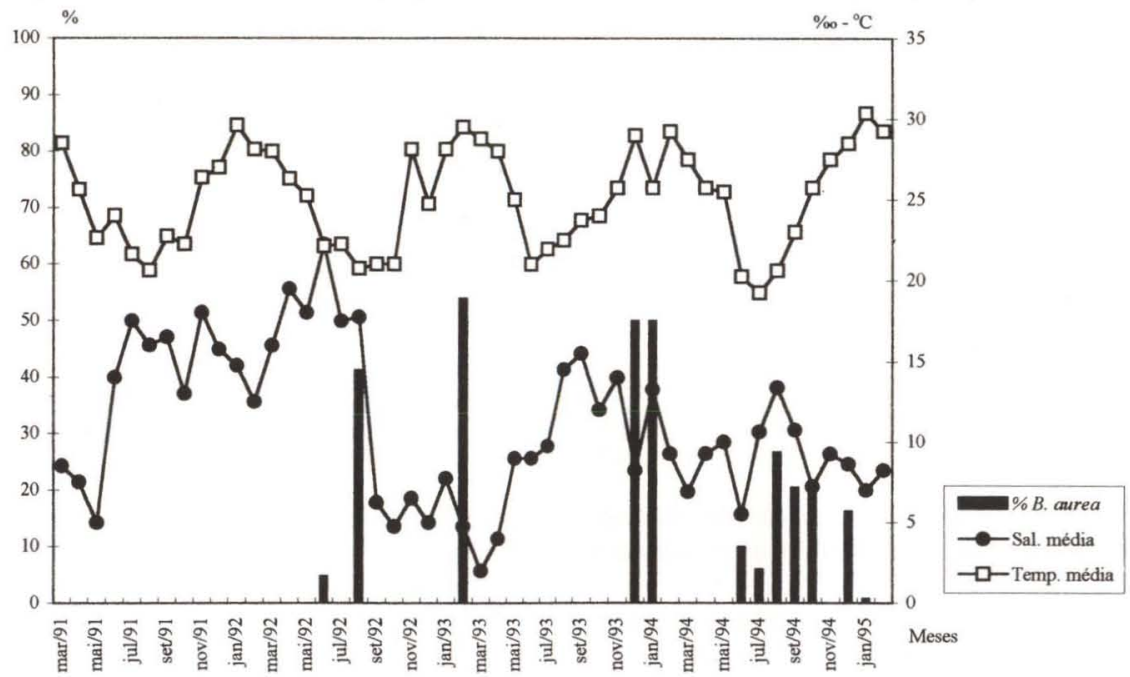

Ano I Ano II Ano III Ano IV

Fig. 2. Distribuição mensal da abundância relativa de Brevoortia aurea em relação à salinidade e temperatura médias na Lagoa Rodrigo de Freitas.

Em relação a distribuição mensal de captura das espécies, verificou-se que Brevoortia aurea (Fig. 2) apresentou freqüência de ocorrência bastante irregular. No Ano I não foi capturada. Nos Anos II e III, as freqüências de ocorrência foram de $25 \%$ e $16 \%$ respectivamente. No Ano IV, esta espécie apresentou um aumento 
significativo de ocorrência, com $58,3 \%$. As maiores abundâncias relativas variaram entre valores médios de salinidade de 5 e $20 \%$. Quanto à temperatura, verificou-se que as maiores abundâncias foram nos períodos em que as temperaturas médias foram superiores a $25^{\circ} \mathrm{C}$.

Para Brevoortia pectinata (Fig. 3), os Anos I e II apresentaram freqüência de ocorrência de $100 \%$ e $75 \%$, respectivamente. No Ano III, esta espécie não foi capturada, enquanto que no Ano IV, sua freqüência de ocorrência foi de $50 \%$, com pico em maio de 1994. Observou-se relativa uniformidade nas abundâncias relativas no Ano I, quando as médias de salinidade variaram em torno de 15 a $20 \%$ e as da temperatura entre 20 e $30^{\circ} \mathrm{C}$. No Ano III, os maiores picos de abundância relativa ocorreram em águas com salinidade de $10 \%$ e temperatura acima de $25^{\circ} \mathrm{C}$.

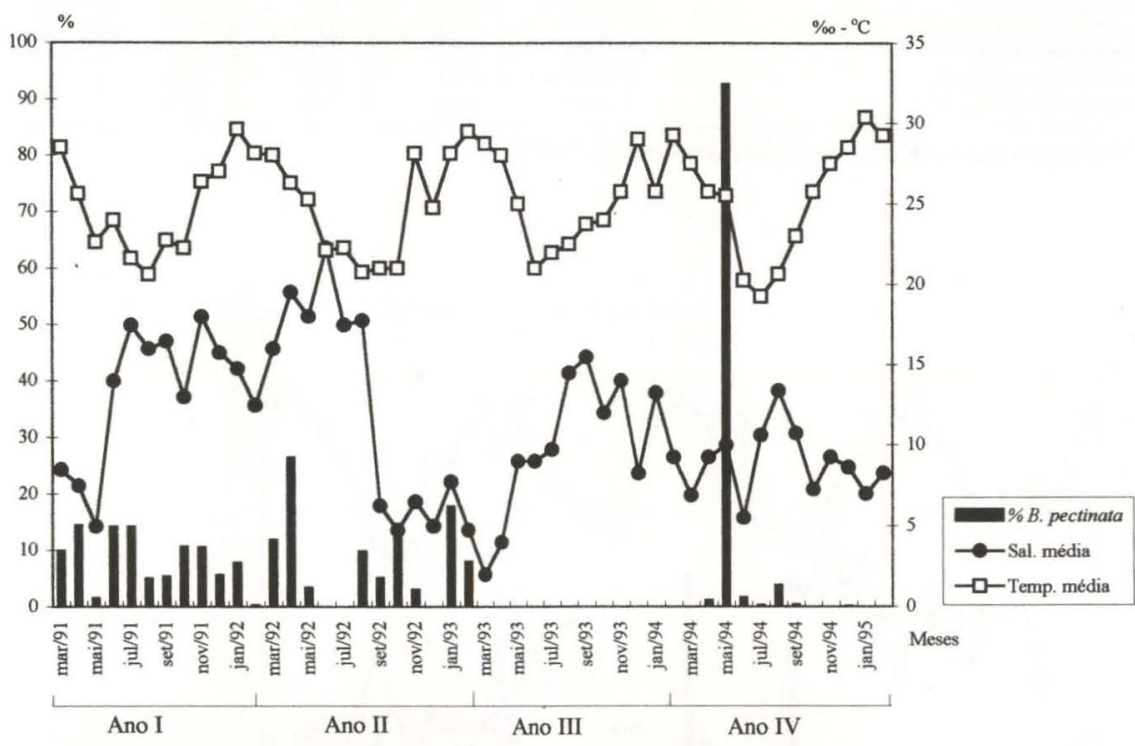

Fig. 3. Distribuição mensal da abundância relativa de Brevoortia pectinata em relação à salinidade e temperatura médias na Lagoa Rodrigo de Freitas.

Em·Genidens genidens a freqüência de ocorrência foi bastante irregular durante todo o período amostrado, com $50 \%$ no Ano I, 58,3\% no Ano II e 8,3\% no Ano III, enquanto que no Ano IV, não foi capturada. Esta distribuição foi caracterizada por grandes picos na abundância relativa em salinidade médias variando entre 5 e $18 \%$, e temperaturas médias, na maioria das vezes, acima de $25^{\circ} \mathrm{C}$ (Fig. 4).

Em relação a Geophagus brasiliensis, a freqüência de ocorrência de $91,6 \%$ no Ano I, $83,3 \%$ no Ano II e de $100 \%$ nos Anos III e IV. A distribuição mensal da abundância relativa foi razoavelmente pouco oscilante, com um padrão de distribuição relativamente bem definido durante todo o período amostrado. As maiores abundâncias ocorreram, geralmente, nos períodos em que as salinidades médias foram inferiores a $10 \%$, com temperaturas médias em torno de 25 a $30^{\circ} \mathrm{C}$ (Fig. 5). 


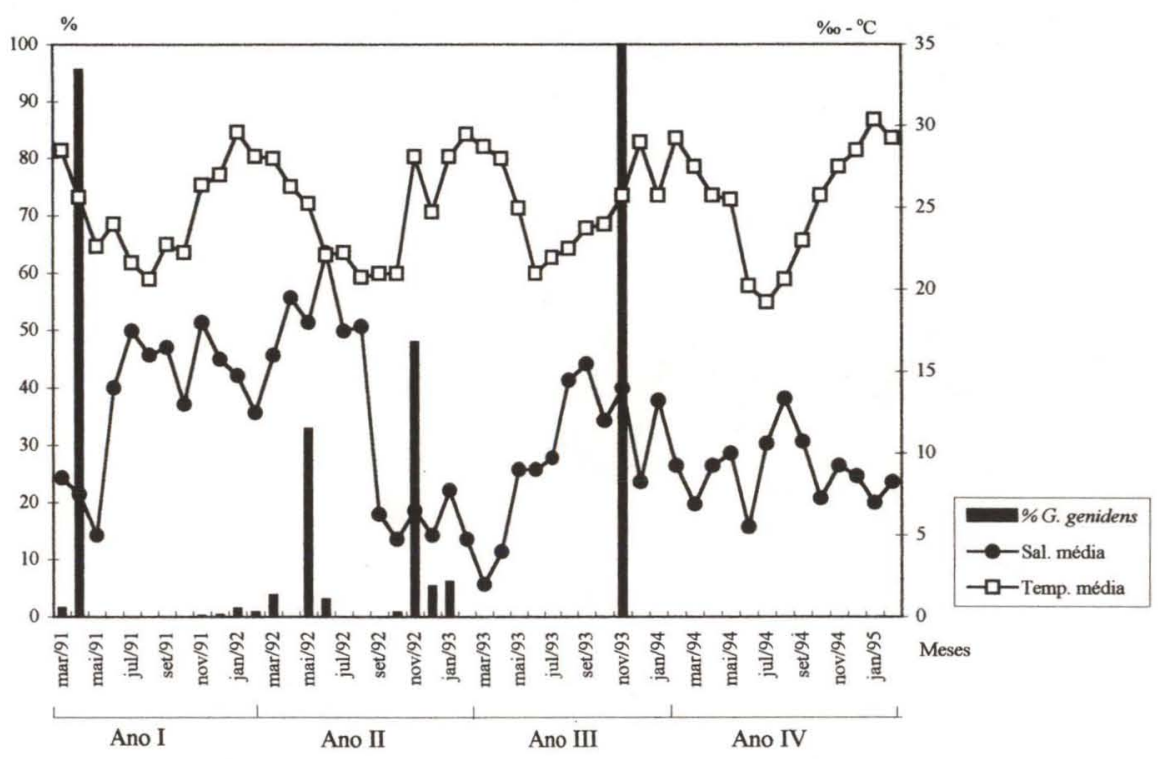

Fig. 4. Distribuição mensal da abundância relativa de Genidens genidens em relação à salinidade e temperatura médias na Lagoa Rodrigo de Freitas.

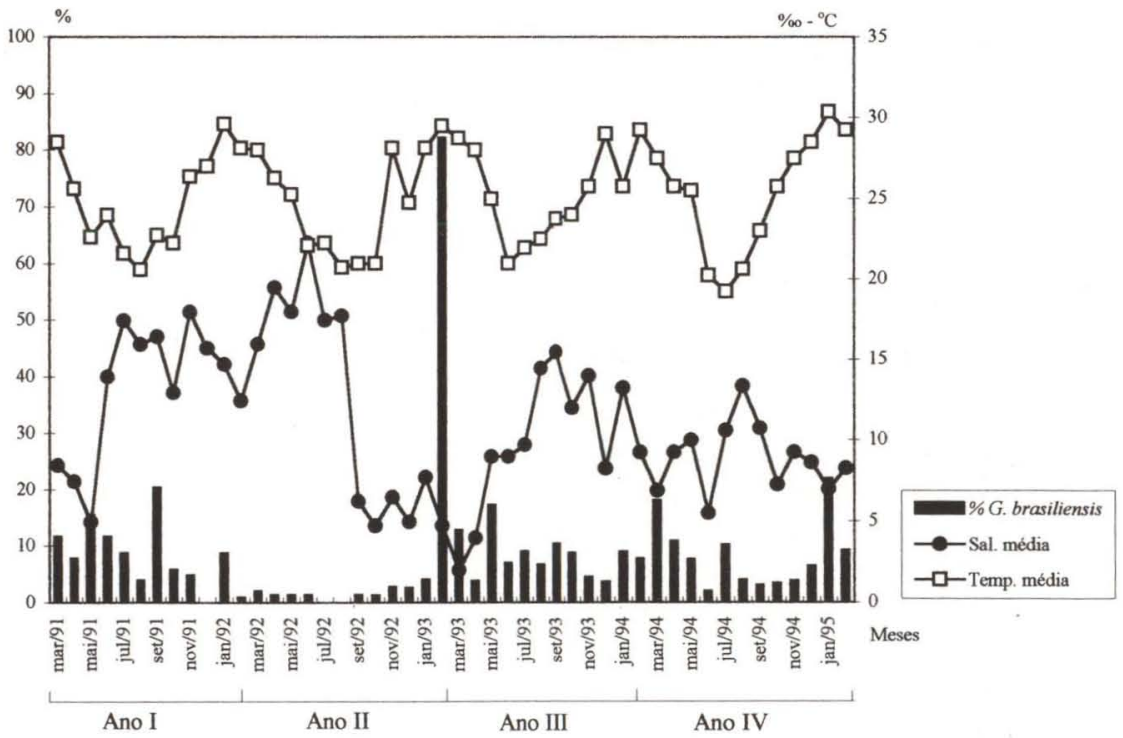

Fig. 5. Distribuição mensal da abundância relativa de Geophagus brasiliensis em relação à salinidade e temperatura médias na Lagoa Rodrigo de Freitas. 
Gerres aprion apresentou freqüência de ocorrência de $100 \%$ nos Anos I e II, $91,6 \%$ no Ano III, e 75\% no Ano IV. A abundância relativa apresentou determinado padrão na distribuição mensal, sendo as maiores abundâncias ocorridas nos períodos em que a salinidade média variou entre 10 e $15 \%$, com temperaturas médias entre 20 e $27^{\circ} \mathrm{C}$ (Fig. 6) .

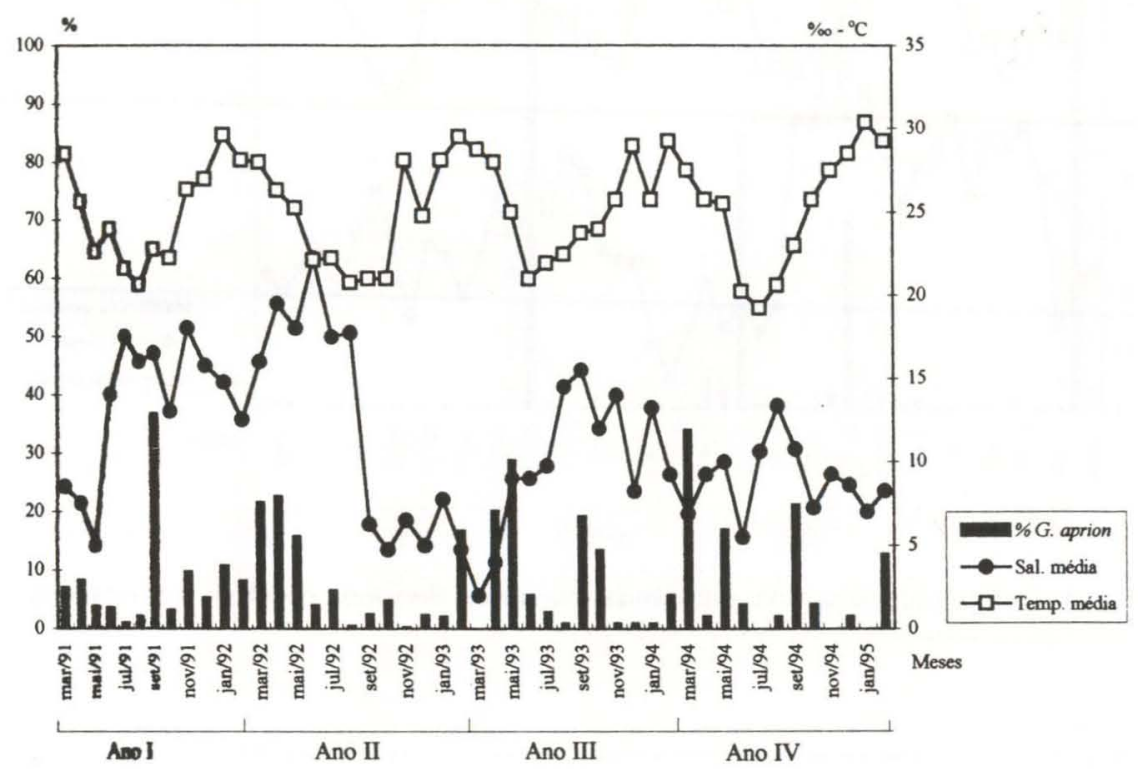

Fig. 6. Distribuição mensal da abundância relativa de Gerres aprion em relação à salinidade e temperatura médias na Lagoa Rodrigo de Freitas.

Para Jenynsia lineata (Fig. 7) a freqüência de ocorrência foi de $100 \%$ em todos os Anos, com uma tendência das maiores abundâncias relativas ocorrerem, geralmente, nos períodos em que os valores médios da temperatura variaram entre 25 e $30^{\circ} \mathrm{C}$, e os valores médios de salinidade foram inferiores a $10 \%$, com exceção do Ano I, quando as salinidades médias foram quase sempre acima de $12 \%$.

Mugil liza apresentou $100 \%$ de freqüência de ocorrência nos Anos I, II e III, e 91,6\% no Ano IV. As maiores abundâncias relativas apresentaram uma tendência geral de se concentrarem entre julho e outubro, período em que as temperaturas médias variaram de 19 a $26^{\circ} \mathrm{C}$ e as salinidades médias de 10 a $19 \%$ (Fig. 8).

Em Poecilia vivipara (Fig. 9) a freqüência de ocorrência foi de $100 \%$ em todos os Anos. A variação da abundância relativa foi similar a J. lineata, com tendência de concentração das maiores abundâncias nos períodos em que as salinidades médias foram inferiores à $10 \%$, com exceção do Ano I, e as temperaturas médias variaram entre 25 e $30^{\circ} \mathrm{C}$.

Xenomelaniris brasiliensis (Fig. 10) apresentou freqüência de ocorrência de $100 \%$ nos Anos I e II, e 91,6\% nos Anos III e IV. A distribuição mensal das 


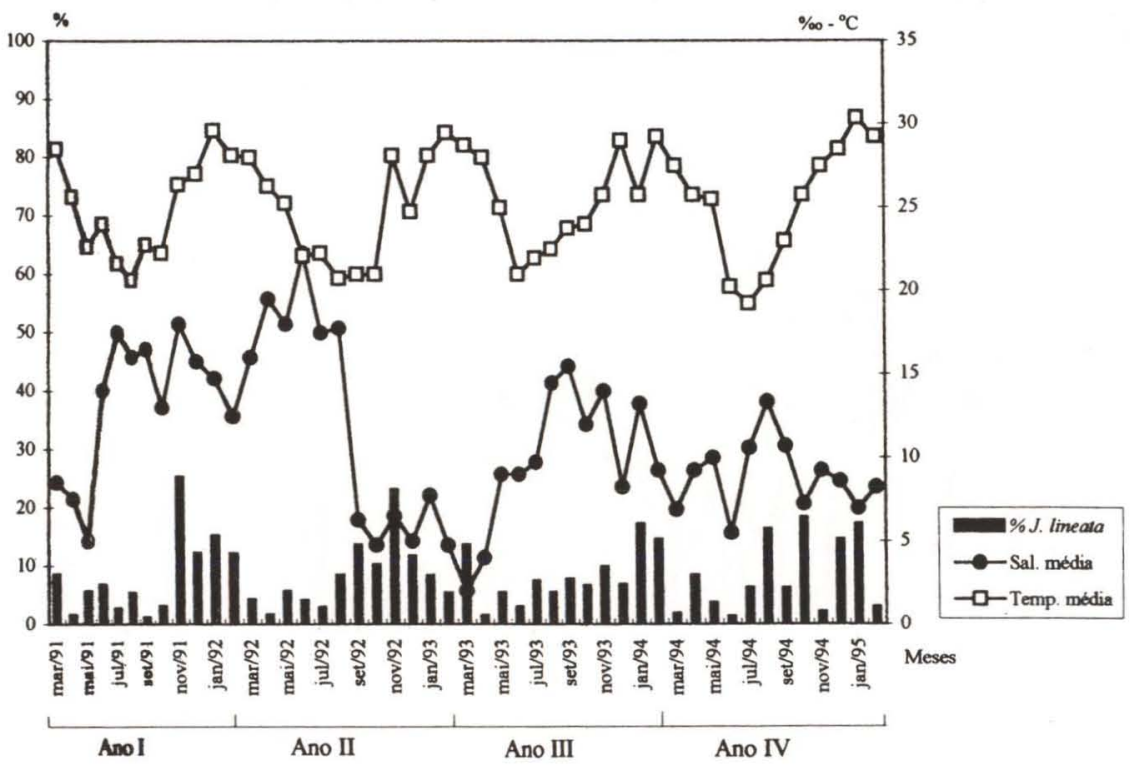

Fig. 7. Distribuição mensal da abundância relativa de Jenynsia lineata em relação à salinidade e temperatura médias na Lagoa Rodrigo de Freitas.

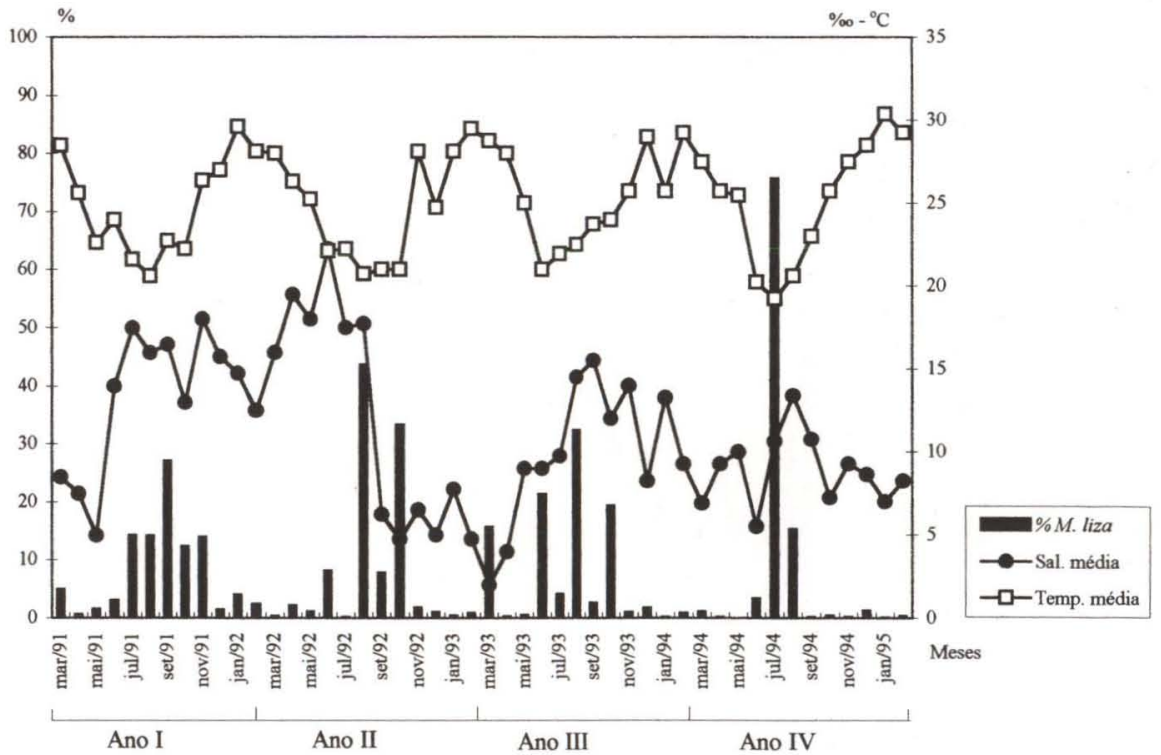

Fig. 8. Distribuição mensal da abundância relativa de Mugil liza em relação à salinidade e temperatura médias na Lagoa Rodrigo de Freitas. 


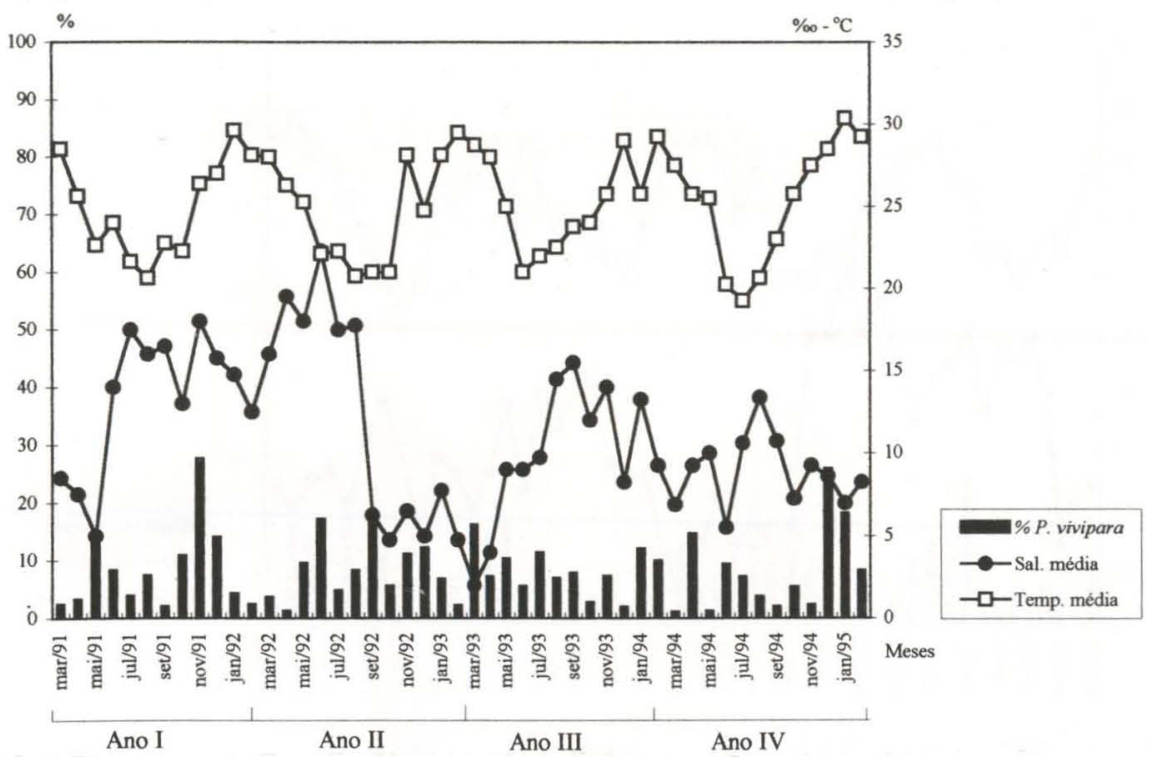

Fig. 9. Distribuição mensal da abundância relativa de Poecilia vivipara em relação à salinidade e temperatura médias na Lagoa Rodrigo de Freitas.

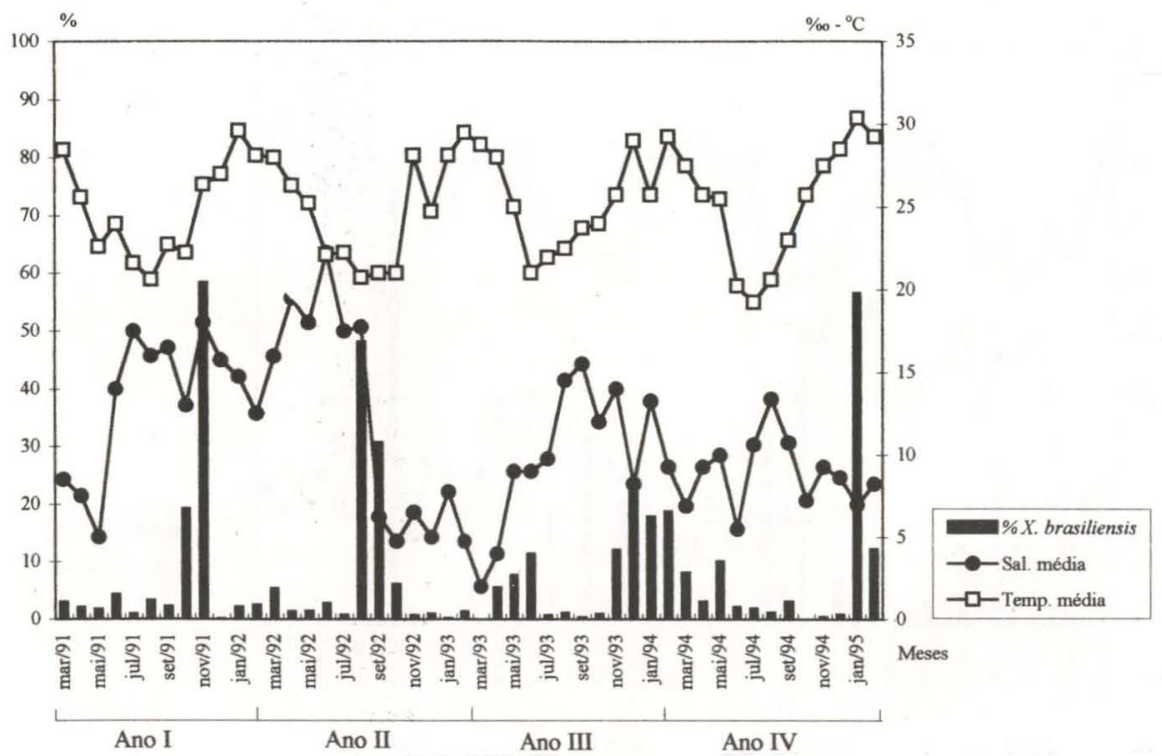

Fig. 10. Distribuição mensal da abundância relativa de Xenomelaniris brasiliensis em relação à salinidade e temperatura médias na Lagoa Rodrigo de Freitas. 
abundâncias relativas foram irregulares, com picos em salinidades médias de 6 a $19 \%$ e temperaturas variando de 20 a $30^{\circ} \mathrm{C}$, não apresentando nenhuma tendência relacionada a estes fatores, porém, nota-se uma gradativa diminuição dos intervalos (meses) entre as maiores abundâncias, no decorrer do período amostrado.

\section{DISCUSSÃO}

Foi capturado na Lagoa Rodrigo de Freitas um número relativamente elevado de espécies, quando comparado aos resultados obtidos por ANDREATA et al. (1990a,b, 1992) para o complexo lagunar da baixada de Jacarepaguá. De maneira geral, a grande maioria das espécies apresentou abundância relativa baixa e ocorrências ocasionais na região.

A comunidade íctica da Lagoa mostrou-se constituída basicamente por espécies marinhas, sendo o número de espécies dulcícolas baixo, mostrando que a composição da ictiofauna apresentou maior influência marinha, enquanto que a influência de aportes fluviais foi baixa, ocorrida principalmente pelo Rio dos Macacos, onde ANDREATA \& MARCA (1993) citaram a ocorrência das espécies de Geophagus brasiliensis, Poecilia reticulata, P. vivipara e Xiphophorus helleri, que também foram coletadas na Lagoa.

Os dados obtidos mostraram que a Lagoa Rodrigo de Freitas é um ambiente com águas salobras, variando de um meio hipotônico a hipertônico, em relação às células animais. Os dois períodos distintos de variação deste fator, provavelmente se relacionam com o sistema de abertura e fechamento das comportas do canal de Jardim de Alah, que é irregular, ou ao assoriamento natural deste canal, que não sofre uma constante dragagem.

A variação verificada na salinidade pode ocasionar problemas osmóticos aos peixes caso eles não apresentem mecanismos eficientes para o controle hídrico, por isso, este ambiente possui uma ictiofauna característica, tendo como principais representantes os peixes eurihalinos.

Considerando-se as variações dos valores da salinidade em relação aos meses do ano, verificamos que ocorre influência direta do tipo de clima da região, que se enquadra no grupo "A" da classificação de Köppen, que define o tipo de clima da região com "AW" (tropical quente-úmido), que é caracterizado por apresentar verão úmido e inverno seco, ocasionando as menores salinidades no verão e as maiores no inverno.

Os valores médios da temperatura superficial variaram de acordo com os meses do ano, refletindo a influência direta da temperatura do ar e da insolação, sendo que no verão estes valores foram relativamente elevados devido a Lagoa ser um ambiente semi-fechado, o que favorece a manutenção do calor em suas águas.

A partir destes pressupostos básicos, podemos relacionar as distribuições das abundâncias relativas das espécies durante o período amostrado.

Em G. brasiliensis, $P$. vivipara e $J$. lineata, que são espécies de origem primária dulcícola, nota-se a tendência geral de aumento nas suas populações nos períodos de menor salinidade e maiores temperaturas, com exceção do Ano I, que devido as condições encontradas, ocorreram picos na abundância em períodos com 
salinidade relativamente alta. Verificou-se que a salinidade foi um fator de maior impacto para $G$. brasiliensis do que para as duas outras espécies dulcícolas, onde foi verificado claramente a limitação da abundância desta espécie por este fator.

Em relação as espécies de origem primária marinha, verifica-se que as espécies do gênero Brevoortia apresentaram uma concentração das maiores abundâncias influenciada principalmente pela temperatura da água, geralmente superiores a $25^{\circ} \mathrm{C}$, enquanto que a salinidade, aparentemente, pouco influenciou, visto a ampla variação da salinidade observada nos picos de abundância destas espécies.

Comparando-se a freqüência de ocorrência e a abundância relativa anual de $B$. pectinata com a de $B$. aurea nota-se claramente uma sucessão entre estas espécies a partir do Ano III, que teve seu início marcado por grande mortandade de peixes, visto que $B$. aurea antes da mortandade era pouco representativa, enquanto que $B$. pectinata era uma das espécies dominantes na região, e posteriormente a esta mortandade houve a inversão deste quadro.

Para Genidens genidens, apesar da baixa freqüência, nota-se um comportando similar as duas espécies de Brevoortia, com tendência aos períodos de águas mais quentes e pouca influência da salinidade em sua distribuição.

Os exemplares de G. aprion, apesar de apresentarem freqüência de ocorrência elevada e presença em uma ampla variação de salinidade e temperatura, apresentaram certa tendência de se concentrarem nas salinidades e temperaturas medianas, porém não há nenhuma razão notável para este comportamento.

Em relação a $M$. liza, que também apresentou uma freqüência de ocorrência elevada, não foi notado nenhuma influência marcante da salinidade na distribuição mensal da abundância, o que enfatiza o seu caráter eurialino, porém verifica-se a tendência desta espécie de se concentrar nos períodos em que os valores da temperatura foram mais baixos.

De maneira geral a salinidade, apesar das espécies estudadas serem consideradas eurialinas, mostrou-se como sendo o fator de maior influência, principalmente para os exemplares das espécies dulcícolas, enquanto que a temperatura foi um fator bastante marcante na abundância das espécies de origem primária marinha, principalmente para B. pectinata, B. aurea, G. genidens e M. liza.

Os resultados do presente trabalho representam uma contribuição para o conhecimento da biologia das espécies estudadas. Dados mais específicos sobre estas espécies e sobre a evolução da Lagoa vêm sendo analisados para futuras divulgações.

É importante ressaltar a necessidade de realizar monitoramento das regiões que vêm sofrendo constante influência antrópica, a fim de evitar que ambientes importantes na preservação das espécies, como a Lagoa, não sejam deixados ao descaso pelas autoridades competentes.

AGRADECIMENTOS. A Profa Dra Jeanete Maron Ramos, Chanceler e chefe de pesquisas da Universidade Santa Úrsula pelo apoio e incentivo às nossas pesquisas. Ao Prof. Philip C. Scott (Universidade Santa Úrsula), pela versão do resumo em inglês, e aos estagiários do Laboratório de Ictiologia (Universidade Santa Úrsula) pelo auxílio nas coletas. 


\section{REFERÊNCIAS BIBLIOGRÁFICAS}

ANDREATA, J.V. 1988. Revisão taxonômica do gênero Diapterus Ranzani, 1840 (Pisces, Perciformes, Gerreidae). Acta. Biol. Leop., São Leopoldo, 10 (1): 59-103.

Gaimard, 1824 (Pisces, Perciformes, Gerreidae) que ocorrem em águas brasileiras. Acta. Biol. Leop., São Leopoldo, 11 (1) : 87-128.

ANDREATA, J.V. \& A.G. MARCA. 1993. Composição dos peixes e macrofauna acompanhante dos riachos e lagos do Parque Nacional da Tijuca e adjacências, Rio de Janeiro, Brasil. Acta Biol. Leop., São Leopoldo, 15 (1): 65-76.

ANDREATA, J.V.; A.M. SAAD \& L.R.R. BARBIÉRI. 1989. Associação e distribuição das espécies de peixes na Laguna de Marapendi, Rio de Janeiro, no período de março de 1985 a fevereiro de 1987. Mem. Inst. Oswaldo Cruz, Rio de Janeiro, 84 (supl. 4): 45-51.

Andreata, J.V.; L.R.R. Barbiéri; A.S.C. Sebília; M.H.C. Silva; M.A.C. SANTOS \& R.P. SANTOS. 1990a. Relação dos peixes da Laguna de Marapendi, Rio de Janeiro, Brasil. Atlântica, Rio Grande, 12 (1): 5-17.

ANDREATA, J.V.; A.M. SAAD; C.R.S.F. BIZERRIL \& F.A. BOCKMANN. 1990 b. Alguns aspectos da ecologia das espécies de peixes da Laguna da Tijuca, período de março de 1987 à fevereiro de 1989. Acta Biol. Leop., São Leopoldo, 12: 247-268.

ANDREATA, J.V; A.M. SAAD; L.A.F. MorAES; C.L. SoARES \& A.G. MARCA. 1992. Associações, similaridade e abundância relativa dos peixes da Laguna de Jacarepaguá, Rio de Janeiro, Brasil. Bol. Mus. Nac. Zool., Rio de Janeiro, 355 : $1-25$.

ARAGÃo, H.R.; J.C.N. PENIDO; M.F. SANTOS \& H.P.L. OlIVEIRA. 1939. Relatório sobre a situação da Lagoa Rodrigo de Freitas sob o ponto de vista biológico. Mem. Inst. Oswaldo Cruz, Rio de Janeiro, 34 (4): 457-463.

BRITO, I.M. \& E.E. LEMOS. 1982. Evolução geológica e fauna da Lagoa Rodrigo de Freitas, Rio de Janeiro. An. Acad. Brasil. Ciênc., Rio de Janeiro, 54(1): 143-164.

Figueiredo, J.L. \& N.A. Menezes. 1978. Manual de Peixes Marinhos do Sudeste do Brasil. II - Teleostei (1). São Paulo, Museu de Zoologia, Universidade de São Paulo, 110p. 1980. Manual de Peixes Marinhos do Sudeste do Brasil. III-Teleostei (2). São Paulo, Museu de Zoologia, Universidade de São Paulo, 90p.

FISCHER, W. 1978. FAO species identification sheets for fishery purposes. Western Central Atlantic (fishing area 31). Rome, Food and Agriculture Organization of the United Nations, vols I-V.

FEEMA (Fundação Estadual de Engenharia do Meio Ambiente). 1975. Lagoa Rodrigo de Freitas. Estudos para o projeto de recuperação. Rio de Janeiro, Feema, Relatório Técnico, 136p.

Godoy, M.P. 1987. Peixes do Estado de Santa Catarina. Florianópolis, Editora da Univ. Fed. Santa Catarina, 572p. 
Lema, T.; C.A.S. Lucena; S. Saenger \& M.F.T. Oliveira. 1979. Primeiro levantamento dos tetraodontiformes do extremo sul do Brasil, Uruguai e Argentina (Teleostei, Acanthopterygii). Comun. Mus. Ciênc. PUCRGS, Porto Alegre, 20: 1-84.

Menezes, N.A. \& J.L. Figueiredo. 1980. Manual de Peixes Marinhos do Sudeste do Brasil. IV-Teleostei (3). São Paulo, Museu de Zoologia, Universidade de São Paulo, 96p.

1985. Manual de Peixes Marinhos do Sudeste do Brasil. V -Teleostei (4). São Paulo, Museu de Zoologia, Universidade de São Paulo, 105p.

MirandA, L.B. 1984. Cinemática e dinâmica de Estuários. São Paulo, B.S.P., $360 \mathrm{p}$.

OLIVEIRA, J.A. 1976. Contribuição ao conhecimento da fauna da Lagoa Rodrigo de Freitas. Rio de Janeiro, Sudepe/RJ, 11 p.

OLIVEIRA, L.P.H. 1955. Sobre a lei da concentração das lagunas e sua aplicação no caso da Lagoa Rodrigo de Freitas. Mem. Inst. Oswaldo Cruz, Rio de Janeiro, 53 (2-4): 264-276.

PIRES, F.R.M. 1977. Solução para o problema ambiental da Lagoa Rodrigo de Freitas, Rio de Janeiro. Rev. Clube de Engenharia, Rio de Janeiro, 408: 43-44.

SALlES-ANDRADE, H.A. 1973. Conclusões químicas sobre as razões da ocorrência de mortandades súbitas na Lagoa Rodrigo de Freitas com base no balanço materiais. Tese de Doutorado, não publicada, Pontifícia Universidade Católica do Rio de Janeiro, Rio de Janeiro, 78p.

VOLCKER, C.M. \& J.V. ANDREATA. 1982. Levantamento taxonômico preliminar da ictiofauna da Laguna da Tijuca, Rio de Janeiro. Rev. Nordest. Biol., João Pessoa, 5 (2): 197-257.

Whitehead, P.J.P.; G.J. Nelson \& T. Wongratana. 1988. FAO species catalogue. Clupeoid fishes of the world (Suborder Clupeoidei). An annotated and illustrated catalogue of the herrings, sardines, pilchards, sprats, anchovies and wolf-herrings. Part 2-Engraulididae. FAO Fish. Synop., Rome, 7 (125): 305-579.

Recebido em 09.V.1996; aceito em 16.IV.1997. 